\title{
Detection of zones of soil contamination with contrast-in-resistivity ecotoxicants by profiling
}

\author{
Evgeniy Shabanov ${ }^{1 *}$, Dmitriy Sirota ${ }^{1}$, and Sergey Prostov ${ }^{1}$ \\ ${ }^{1}$ T.F. Gorbachev Kuzbass State Technical University, Str. Vesennyaya 28, Kemerovo, Russian \\ Federation, 650000
}

\begin{abstract}
A method for detecting zones of soil contamination with contrast-in-resistivity ecotoxicants, as well as geophysical methods for monitoring soil decontamination processes, are discussed in the article. A zone of soil contamination was designed and simulated using electrical prospecting methods - sounding and profiling. The direct and inverse problem of electrical prospecting of a contaminated zone in the form of a sphere inside a homogeneous soil body was solved in this work, while the main design dependencies were determined, which most accurately describe the data obtained experimentally. The proposed algorithm, based on adaptive step size random search, allows to quickly determine the depth and the effective radius of a zone of contamination with contrast-inresistivity ecotoxicants, based on the results of profiling, and to estimate the degree of pollution by the ratio of the resistivity of the anomalous zone to that of the host medium.
\end{abstract}

\section{Introduction}

Environmental protection is the most pressing problem of our time [1-3]. Over time, due to population growth and industrial development, the pressure on the environment increases and pollutants accumulate in water, soil and atmosphere. This accumulation is a negative phenomenon and can lead to harm to the life and health of citizens. At the same time, pollution of the atmosphere and water leads directly to harm to flora, fauna and humans, and soil pollution in the long term leads to negative consequences for plants, animals and humans.

The development of methods for monitoring the environment is one of the promising fields in science [4-6], which will make it possible to more quickly and accurately determine the degree and location of pollution, which has a positive effect on the state of the environment. Direct methods are used for soil monitoring, which involve the extraction of soil samples and their study in laboratory conditions, which is a laborious process [7-8]. In this situation, the development of indirect contactless and not requiring well logging methods of monitoring, such as geophysical methods [9-10], which make it possible to quickly detect the contamination zone by electrical sounding and resistivity profiling, is relevant. These methods are applicable for contrast-in-resistivity pollutants that have anomalously high or low electrical resistivity compared to that of a natural aqueous solution

* Corresponding author: shabanovea@kuzstu.ru 
that fills the soil space. Contrast-in-resistivity ecotoxicants include oil products, solutions of acids and alkalis [11-12]. Due to this property, they are easily and accurately detected in the soil, and measurements of the true electrical resistivity allow quantitative determining the degree of soil contamination with oil products, using a previously developed algorithm. [13]. A method for rapid monitoring of soil pollution and oil product decontamination was developed at KuzSTU, described in detail in the works [14-15].

The purpose of this work is to develop an algorithm based on adaptive step size random search, which makes it possible to quickly detect the zone of contamination with contrastin-resistivity ecotoxicants based on the resistivity profiling data.

\section{Research methods}

In most cases, the contamination zone can be approximated by a spherical inclusion with anomalous resistivity $(\mathrm{R})$.

Consider the solution of the direct problem of electrical prospecting for this case: a conducting sphere with radius $a$ and resistivity $\rho_{2}$ is located in a homogeneous isotropic half-space with resistivity $\rho_{1}$ at a certain depth $h$. A three-electrode device AMN is located on the earth's surface (electrode B is infinite): where A is a current electrode and $\mathrm{MN}$ are potential electrodes. The distance from the center of the sphere $\mathrm{Q}$ to the point $\mathrm{A}$ is $\mathrm{d}, \mathrm{m}$; $\mathrm{a}$ to the potential measuring point $\mathrm{M}-\mathrm{r}, \mathrm{m}$; the electrode half-spacing - L, m (Fig. 1).

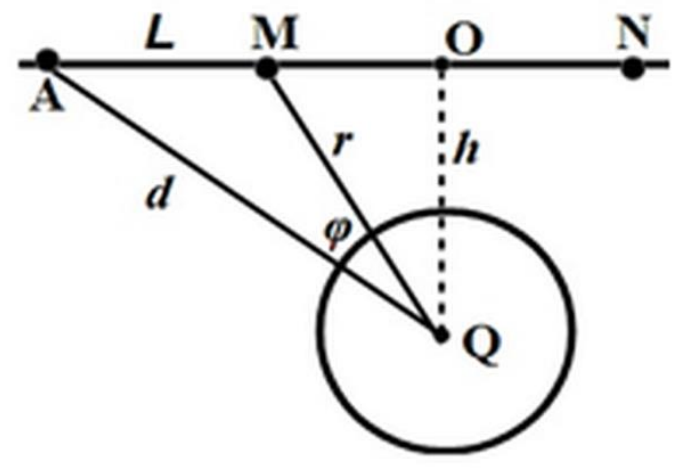

Fig. 1. Calculation pattern

Set the polar coordinate system $r=r(\varphi)$ with the origin at the center of the sphere $\boldsymbol{Q}$ and the polar axis $A Q$. In such a coordinate system, the Laplace equation for the potential $\boldsymbol{u}$ will be of the form of (1) - (4):

$$
\frac{\partial}{\partial r}\left(r^{2} \frac{\partial u}{\partial r}\right)+\frac{1}{\sin \varphi} \frac{\partial}{\partial \varphi}\left(\sin \varphi \frac{\partial u}{\partial \varphi}\right)=0 .
$$

Solutions to the equation outside the sphere $u_{e}=\frac{I \rho_{1}}{4 \pi L}+u_{1}(r, \varphi)$ and inside the sphere $u_{i}=\frac{I \rho_{1}}{4 \pi L}+u_{2}(r, \varphi)$ must meet the following boundary conditions:

$$
\begin{gathered}
\frac{\partial u_{1}(a, \varphi)}{\partial r}=\frac{\rho_{1}}{\rho_{2}} \cdot \frac{\partial u_{2}(a, \varphi)}{\partial r} \\
u_{1}(a, \varphi)=u_{2}(a, \varphi)
\end{gathered}
$$




$$
u_{1}(\infty, \varphi)=0
$$

The general solution of the equation (1), taking into account conditions (2), will be the following expression:

$$
u_{e}=\frac{I \rho_{1}}{2 \pi}\left[\frac{1}{L}+2 \sum_{j=1}^{\infty} \frac{K_{j} a^{2 j+1}}{d^{j+1} r^{j+1}} P_{j}(\cos \varphi)\right]
$$

where $K_{j}=\frac{j(q-1)}{(J+1) q+1}-$ reflection coefficient; $q=\frac{\rho_{2}}{\rho_{2}} ; P_{j}(\cos \varphi)-$ Legendre polynomials.

Determining the intensity $\boldsymbol{E}$ in terms of the derivative $\frac{\partial u_{e}}{\partial L}$ and applying the formula for calculating the resistivity $\rho_{k}=\frac{E}{I} 2 \pi L^{2}$, we obtain the following formula:

$$
\rho_{k}=\rho_{1}\left[1+2 L \sum_{j=1}^{\infty} \frac{K_{j} a^{2 j+1}(j+1)}{d^{j+1} r^{j+2}}\left\{r P_{j}(\cos \varphi)-d P_{j+1}(\cos \varphi)\right\}\right]
$$

where the relationship between the distances $\boldsymbol{d}, \boldsymbol{r}$, the polar angle $\boldsymbol{\varphi}$ and the coordinates of the points $\boldsymbol{x}_{\boldsymbol{A}}, \boldsymbol{x}_{M}$ relative to the projection of the center of the sphere are determined by the following formulas:

$$
\begin{gathered}
r^{2}=x_{M}^{2}+h^{2}, \\
d^{2}=x_{A}^{2}+h^{2}, \\
\cos \varphi=\frac{r^{2}+d^{2}-L^{2}}{2 r d} \\
x_{A}=x_{M}-L
\end{gathered}
$$

From formula (4), an expression for the resistivity in the half-space surrounding the sphere, which is in a uniform electric field generated by the infinite electrode, can be obtained in rectangular coordinates:

$$
\rho_{k}=\rho_{1}\left[\frac{2 K_{1} a^{3}\left(h^{2}-2 x_{M}^{2}\right)}{\left(h^{2}+x_{M}^{2}\right)^{5 / 2}}\right]
$$

Pass on to solving the inverse problem.

Suppose that electrical profiling has been performed on the surface of the earth, as a result of which the resistivity distribution is determined, the graph of which allows making an assumption about the presence of some anomaly in the lower part of the half-space. It is required to find the values of the following three parameters: the sphere depth $-h, \mathrm{~m}$; the sphere radius - $a, \mathrm{~m}$; the value of the ratio of the resistivity of the sphere to that of the surrounding medium - $q$. We will use the values $\rho_{k}^{j}$ at three extreme points of the curve (left, center, right) as initial information. Thus, it is required to solve a system of three nonlinear equations in three unknowns of the following form:

$$
\rho_{K}^{j}=\rho\left(a, h, q, x_{M}^{j}\right)
$$

where $x_{M}^{j}, j=1,2,3$ - coordinates of measurement points of the resistivity curve; $\rho_{K}^{j}-$ 
measured at these points of the resistivity curve.

To reduce the number of variables to be determined, we use expression (6). Find the derivative from it and equate it to zero:

$$
\frac{\partial \rho_{k}}{\partial x_{M}}=\frac{2 \rho_{1} K_{1} a^{3}\left(6 x_{M}^{3}-9 x_{M} h^{2}\right)}{\left(h^{2}+x_{M}^{2}\right)^{7 / 2}}=0 .
$$

It can be seen that the coordinates of the extreme points are determined only by the depth of the sphere center. Then the estimated value for the depth of the sphere is determined by the formula:

$$
h=\frac{\sqrt{6}}{3} x_{M}
$$

Since the experimental curve of electrical profiling may not be symmetrical, the arithmetic mean of the depths calculated from the left and right extreme points can be taken as an approximate depth.

Thus, it is required to select only two parameters: the sphere radius - $a \in(0 ; h), \mathrm{m}$, as well as the value of the ratio of the resistivity of the sphere to that of the surrounding medium $-q \geq 0$. The algorithm for determining the required parameters is shown in Fig. 2 .

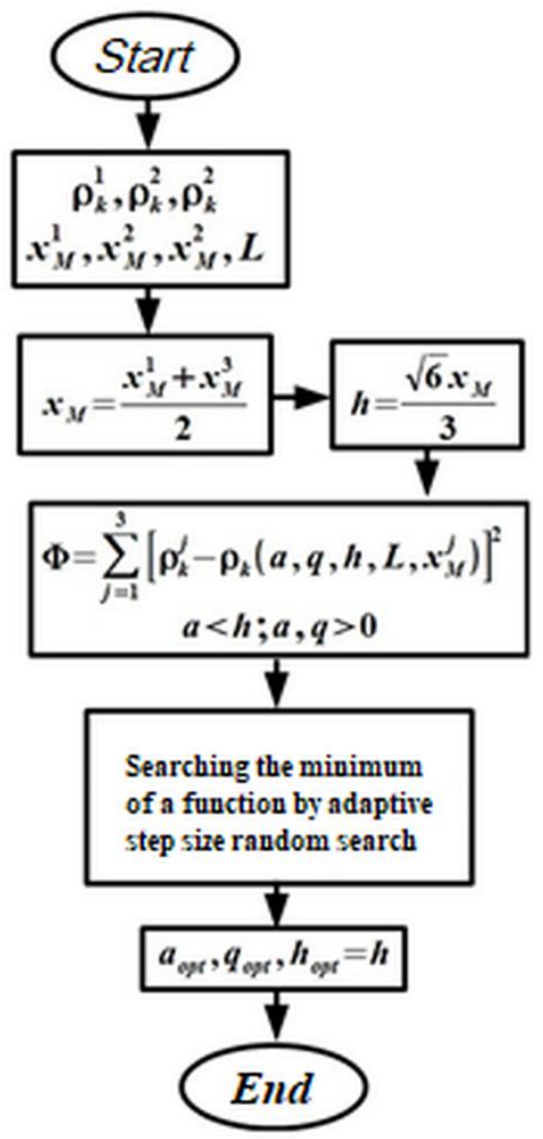

Fig. 2. Inversion algorithm 
We will solve the newly defined system of three equations (7) using the least squares method. Compose the corresponding objective function with obvious constraints to variables of the form $a, h, q>0 ; h>a$ :

$$
\Phi(a, q)=\sum_{j=1}^{3}\left[\rho_{k}^{j}-\rho\left(a, h, q, x_{M}^{j}\right)\right]^{2} \rightarrow \min .
$$

Analysis of the function surface (9) at various depths $\boldsymbol{h}$ and the electrode half-spacing $L$ shows that this function is unimodal and has a ravine structure. For searching its minimum, adaptive step size random search can be used (5). The advantage of this method is that there is no need to calculate the partial derivatives of the objective function, as well as confident movement along the bottom of the ravine towards the extreme point.

\section{Results and discussion}

Consider a number of cases for the use of the technique for determining the required parameters. Experimental data were obtained from geoelectrical monitoring of clay rock reinforcement zones.

The results of resistivity profiling of the flooded zone of burnt coal, section 4 Vnutrenny at the Yuzhno-Abinsky site "Podzemgaz" (see Fig. 3).

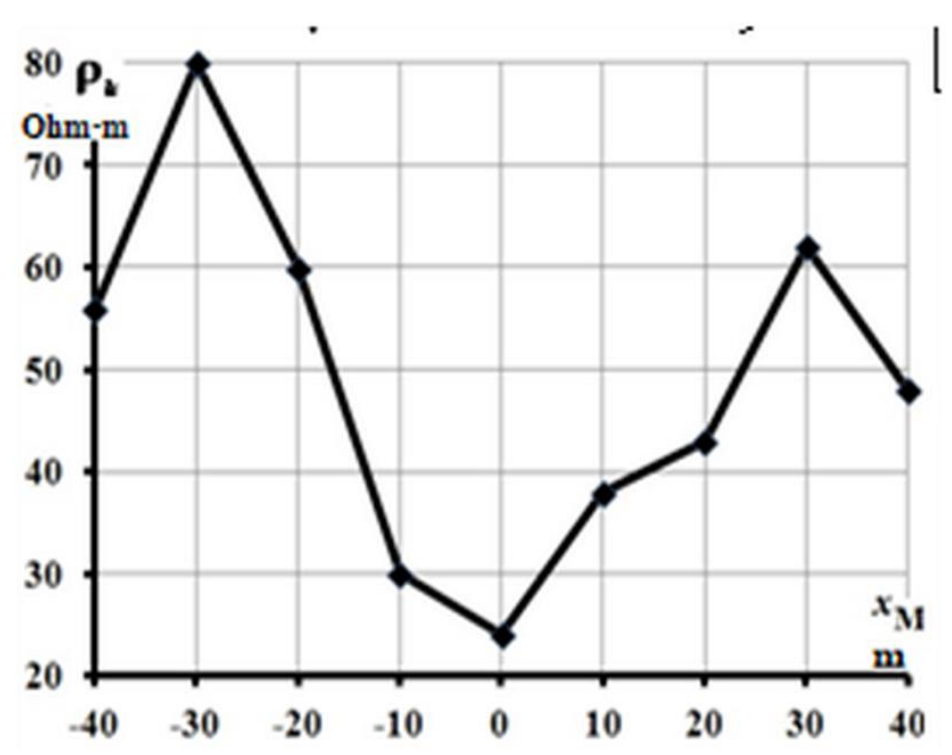

Fig. 3. Results of resistivity profiling at section 1

$$
\rho_{1}=56, \rho_{\mathrm{k}}^{1}=80, \rho_{\mathrm{k}}^{2}=24, \rho_{\mathrm{k}}^{3}=62 \text { OM } \cdot \mathrm{M} ; \mathrm{x}_{\mathrm{M}}^{1}=-30, \mathrm{x}_{\mathrm{M}}^{2}=0, \mathrm{x}_{\mathrm{M}}^{3}=30 \mathrm{M}
$$

- the extreme values of the results of resistivity profiling of the flooded area [6].

Since the coordinate of the right extreme point is equal to $x_{M}=30$, the approximate depth is:

$$
h=\frac{\sqrt{6}}{3} x_{M}=10 \sqrt{6} \approx 24,5 \mathrm{M} .
$$

Since the measurements spacing are unknown, we will compile a table of possible values of the radii and resistivity ratios for different $A M$ half-spacings. 
Table 1. Possible values of the radii and resistivity ratios

\begin{tabular}{|l|l|l|l|l|}
\hline $\mathrm{AM}, \mathrm{m}$ & 15 & 25 & 35 & 45 \\
\hline$a, \mathrm{~m}$ & 21.6 & 24.5 & 24.5 & 24.5 \\
\hline$q$ & 0.32 & 0.61 & 0.49 & 0.44 \\
\hline
\end{tabular}

It should be noted that with an increase in the $A M$ half-spacing (i.e., when $A M$ tends to infinity), the radius of the assumed sphere reaches its possible maximum $\left(a_{\max }=h\right)$, and the resistivity ratio tends to zero $(q \rightarrow 0)$. Thus, the assumed radius of the sphere is in the interval $a \in[21 ; 22] \mathrm{m}$, and the resistivity ratio is in the interval $a \in[0,3 ; 0,4]$.

The results of resistivity profiling of the flooded zone of the bulkhead of the hydraulic spoil bank No 3 of the Kedrovsky open-pit mine (Fig. 4): $\rho_{1}=30, \rho_{\mathrm{k}}^{1}=38, \rho_{\mathrm{k}}^{2}=10, \rho_{\mathrm{k}}^{3}=$ $38 \mathrm{Ohm} \cdot \mathrm{m} ; \mathrm{x}_{\mathrm{M}}^{1}=-15, \mathrm{x}_{\mathrm{M}}^{2}=0, \mathrm{x}_{\mathrm{M}}^{3}=13 \mathrm{~m}$ - the extreme values of the results of resistivity profiling of the flooded zone.

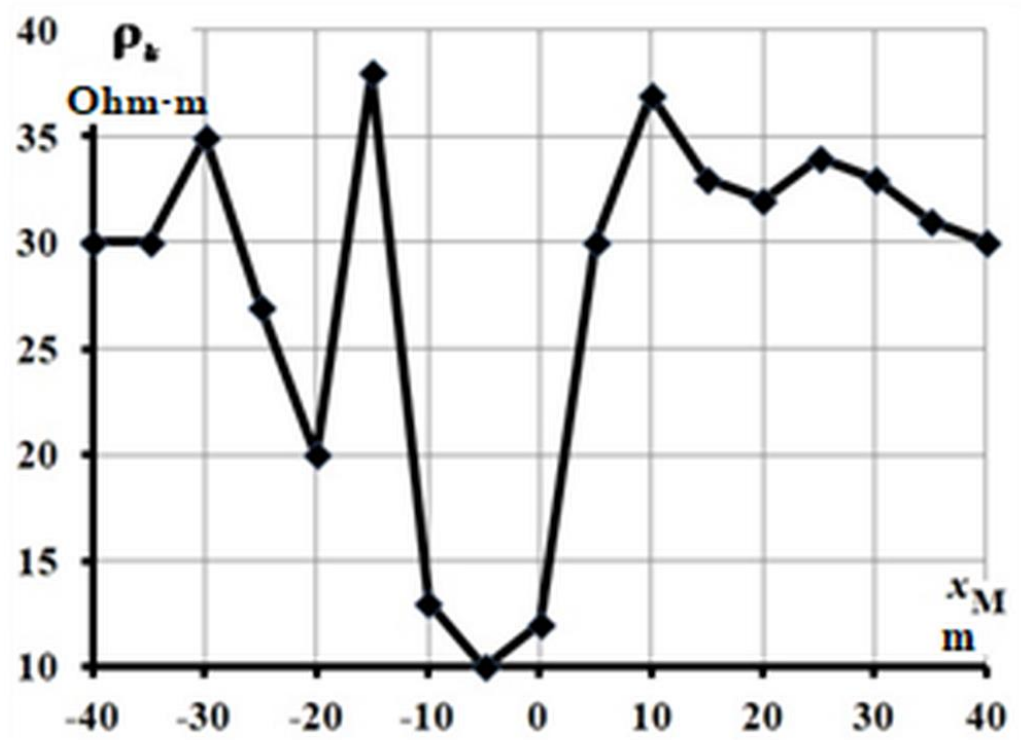

Fig. 4. Results of resistivity profiling at section 2 (profile No. 1)

Since the coordinates of the left and right extreme points are not symmetrically located relative to the center, the anomalous resistivity area can only approximately be considered a sphere. We will consider the arithmetic mean calculated from the left point $x_{M}=15$, for which the depth is $h_{1}=\frac{\sqrt{6}}{3} x_{M} \approx 12,3 \mathrm{~m}$, and the right point, for which the depth is $h_{2}=$ $\frac{\sqrt{6}}{3} x_{M} \approx 10,6 \mathrm{~m}$, as the approximate depth of the center of the area.

Thus, the approximate depth is $h=11.45 \mathrm{~m}$. These resistivity profiling values were obtained at a half-spacing of $A M=30 \mathrm{~m}$. The results of solving the inverse problem show that the anomaly radius is $a \approx 8,36 \mathrm{~m}$, and the ratio of the surrounding space resistivity to that of the anomalous area is $q \approx 0,0056$, which indicates a higher conductivity of the anomalous zone $\rho_{2}=q \cdot \rho_{1}=0.224 \mathrm{Ohm} \cdot \mathrm{m}$.

At the same test section, the following results of profile No. 2 were obtained: 5).

$\rho_{1}=50, \rho_{k}^{1}=50, \rho_{k}^{2}=65, \rho_{k}^{3}=45 \mathrm{Oм}^{\cdot} \mathrm{M} ; x_{M}^{1}=-20, x_{M}^{2}=0, x_{M}^{3}=25$ м (see Fig. 


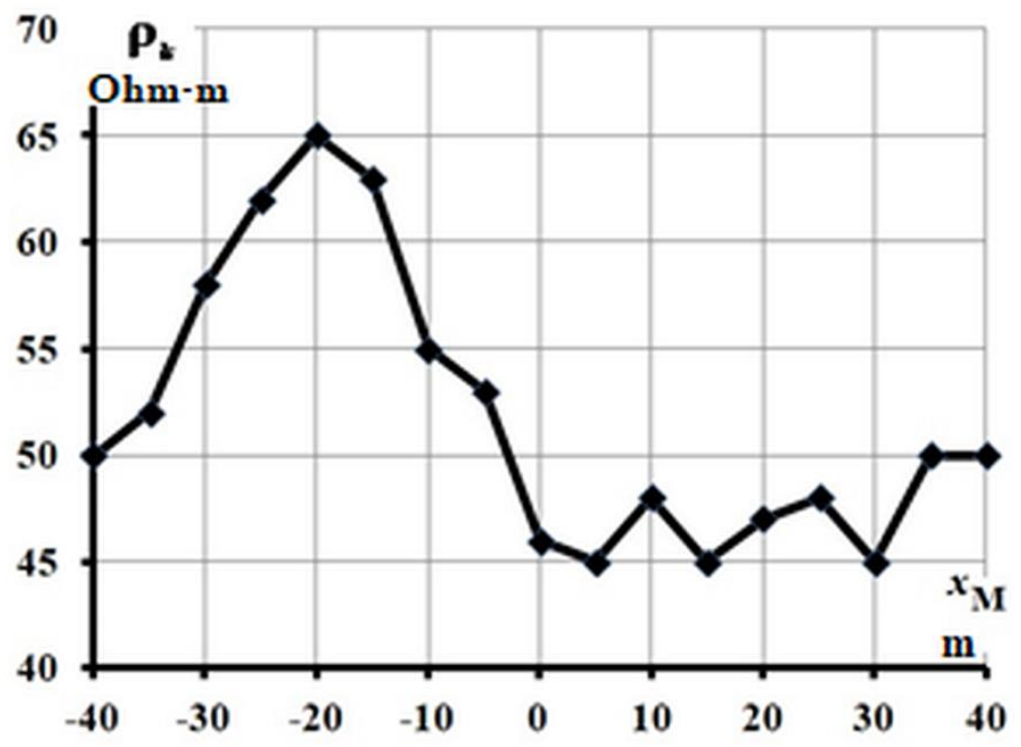

Fig. 5. Results of resistivity profiling at section 2 (profile No. 2)

The coordinates of the left and right extreme points are not symmetrical about the center. We will consider the arithmetic mean calculated from the left point $x_{M}=20$, for which the depth is $h_{1}=\frac{\sqrt{6}}{3} x_{M} \approx 16,3 \mathrm{~m}$, and the right point $x_{M}=25$, for which the depth is $h_{2}=\frac{\sqrt{6}}{3} x_{M} \approx 20,4 \mathrm{~m}$, as an approximate depth of the center of the area. Thus, the approximate depth is $h=11.45 \mathrm{~m}$. These resistivity profiling data were also obtained at a half-spacing of $A M=30 \mathrm{~m}$. The approximate results of solving the inverse problem show that the anomaly radius is $a \approx 18,35 \mathrm{~m}$, and the ratio of the surrounding space resistivity to that of the anomalous area is $q \approx 1,56$. The coincidence of the assumed radius of the sphere with the depth of its center indicates that the presented data do not agree with the calculation pattern used. For example, the first extreme resistivity value should be located more to the left than it is actually located on the graph, while its value should be less than $\rho_{1}=50$.

The results of electrical profiling of the injection reinforcement zone at the OOO NOOCENTR geopolygon (see Fig. 6) are:

$\rho_{1}=16, \rho_{\mathrm{k}}^{1}=17, \rho_{\mathrm{k}}^{2}=8, \rho_{\mathrm{k}}^{3}=20$ OM$\cdot \mathrm{M} ; \mathrm{x}_{\mathrm{M}}^{1}=-3,8, \mathrm{x}_{\mathrm{M}}^{2}=0, \mathrm{x}_{\mathrm{M}}^{3}=4 \mathrm{~m}$

- the extreme values of the results of electrical profiling of the flooded area.

The coordinates of the left and right extreme points are located almost symmetrically relative to the center of the extreme area. Then the approximate depth of the center of the area calculated from the right point $x_{M}=4$ will be equal to $h=\frac{\sqrt{6}}{3} x_{M} \approx 3,26 \mathrm{~m}$. These resistivity profiling values were obtained at a half-spacing of $A M=3 \mathrm{~m}$. The approximate results of solving the inverse problem show that the anomaly radius is $a \approx 3 \mathrm{~m}$, and the ratio of the surrounding space resistivity to that of the anomalous area is $q \approx 0,65$. 


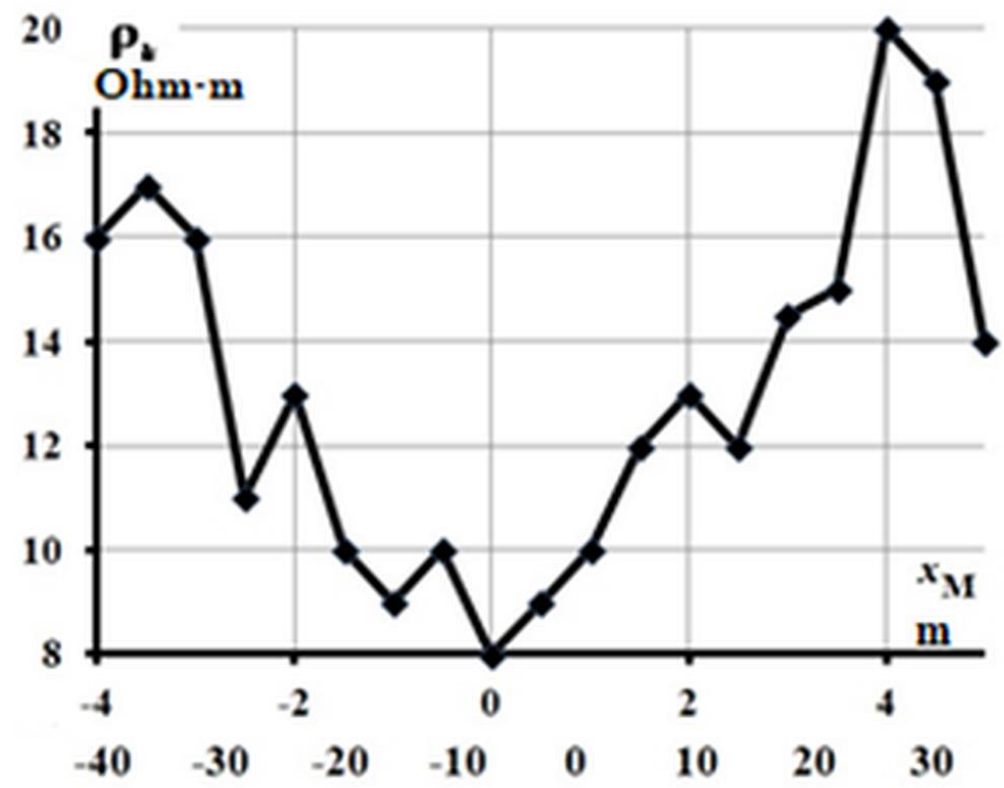

Fig. 6. Results of resistivity profiling at section 3

\section{Conclusions}

Based on the results of the study, the following conclusion can be drawn: the proposed algorithm, based on adaptive step size random search, allows to quickly determine the depth and the effective radius of a zone of contamination with contrast-in-resistivity ecotoxicants, based on the results of profiling, and estimate the degree of pollution by the ratio of the resistivity of the anomalous zone to that of the host medium.

The study was conducted as part of grant MK-1212.2020.5 "Geological and geophysical monitoring of the processes of electrochemical cleaning of soil bases of structures from oil pollution".

\section{References}

1. G. Semenova, E3S Web of Conferences. International Scientific and Practical Conference "Environmental Risks and Safety in Mechanical Engineering" (ERSME2020), 04007 (2020)

2. R. M. Usmanova, N. A. Sattarova, N. N. Boiko, IOP Conference Series: Materials Science and Engineering. International Science and Technology Conference (FarEastCon 2020), 062040 (2021)

3. V. Zakrutkin, G. Sklyarenko, E. Gibkov, O. Reshetnyak, A. Rodina,16th International Multidisciplinary Scientific GeoConference SGEM 2016, 87 (2016)

4. E. A. Lushchay, D. E. Ivanov, E. I. Tikhomirova, Povolzhskiy Journal of Ecology, 4, 458 (2019)

5. E. Loupian, V. Savorskiy, A. Kashnitskii, D. Kobets, K. Sen'Ko, I. Balashov, I. Uvarov, E3S Web of Conferences. Regional Problems of Earth Remote Sensing, 01002 (2020) 
6. Q. Wu, H. Liu, S. Liu, Y. Zeng, M. Zhang, H. Zhao, Meitan Xuebao, 44, 1, 10 (2019)

7. W. M. Badawy, K. Ali, H. M. El-Samman, M. V. Frontasyeva, S. F. Gundorina, O. G. Duliu, Physics of Particles and Nuclei Letters, 12, 4, 637 (2015)

8. M. Imchen, R. Kumavath, D. Barh, V. Avezedo, M. Viana, P. Ghosh, A. R. Wattam, Scientific Reports, 7, 1, 8859 (2017)

A. P. Chernov, A. V. Danilov, S. A. Vyzhva, 18th International Conference "Geoinformatics: Theoretical and Applied Aspects", 16344 (2019)

I. M. Hasanov, L. A. Muravyev, 15th Conference and Exhibition Engineering and Mining Geophysics, 733 (2019)

9. E. Kardasz, P. Kardasz, V. Pohrebennyk, A. Shybanova, N. Homko, 19th INTERNATIONAL SCIENTIFIC GEOCONFERENCE SGEM 2019, 193 (2019)

A. M. Gareev, R. G. Galimova, Journal of International Scientific Publications: Ecology \& Safety, 6, 1, 390 (2012)

10. S. M. Prostov, M. B. Gucal, E. F. Shabanov, Chinese Coal in the XXI Century: Mining, Green and Safety. Taishan Academic Forum - Project on Mine Disaster Prevention and Control, 433 (2014)

11. S. Prostov, E. Shabanov, E3S Web of Conferences. 3rd International Innovative Mining Symposium, IIMS 2018: Electronic edition, 02002 (2018)

12. S. Prostov, E. Shabanov, E3S Web of Conferences. The Second International Innovative Mining Symposium, 02007 (2017) 